\title{
Learnings from Designing 'Simplified' UX for Complex Planning and Scheduling Application for Enterprises
}

\author{
Tanu Malhotra \\ User Experience Designer, SAP Labs \\ Bangalore, India \\ tanu.malhotra@sap.com
}

\author{
Krishnan Vijayaraghavan \\ User Experience Designer, SAP Labs \\ Bangalore, India \\ krishnan.vijayaraghavan@sap.com
}

This paper narrates the journey of redesigning user experience for a complex Enterprise Resource Planning (ERP) Planning and Scheduling Application. Through the medium of this paper, we would also share the challenges and learnings from redesigning the user interface of this planning and scheduling application that is used by experienced power users and new generation users both.

Planning, Scheduling; Gantt Chart; Manufacturing; Enterprise Applications; User Interfaces; User Experience (UX) Design; UX Methodology, Business Software, Case Study, Usability, Usability Testing.

\section{INTRODUCTION}

Production Scheduling Board (PSB) is SAP's ERP application used in the manufacturing process by Production Planners, majorly on desktops or large monitors. PSB is an interactive solution that helps the users (Production Planners) in planning and scheduling of orders with respect to time and resources. By PSB, users create a short-term production plan that matches the overall supply to demand, given available resources and production methodology.

The old PSB application that had been used by customers for more than a decade, was designed and developed on SAP's Universal Graphical User Interface (SAP GUI). This version of the planning and scheduling application that was used by around 300 customers across industries, though being very powerful, proved to have an extremely cumbersome interface.

Hence, it was essential to redesign the old PSB application that would not only be based on the new technology - SAP UI5 but would also meet the needs of new generation and existing users both.

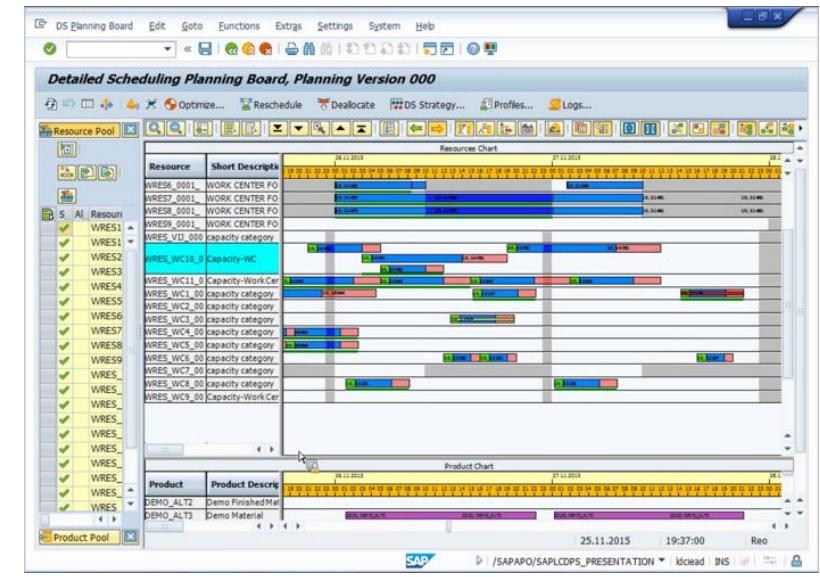

Figure 1: SAP GUI version of Graphical Planning Board

To design the new PSB application, we had to strictly adhere to SAP's user experience guidelines, that were based on SAPUI5, known as SAP Fiori. SAP Fiori highlights simplicity as its core principle and we were required to drive the new PSB application design in this direction. 
In the further sections of this paper, we would present our experiences in designing the Production Scheduling Board.

\section{DISCOVER}

To initiate the process of redesigning an enterprise application that had been in use for more than ten years, it was necessary for us to comprehend its usage, functionalities and shortcomings. This initial information was provided to us by the product development team.

\subsection{Domain understanding}

PSB application caters to manufacturing, which is a complex domain as it encompasses innumerable processes. In this regard, it was crucial for us to get an appreciation of the basic terminologies, processes and subsequent use cases.

\subsection{Customer workshops}

We conducted group workshops with customers using the approach of design thinking, a methodology to solve complex problems using creative techniques, which helped them articulate their needs, expectations and pain points.

\subsection{Expert feedback}

The users we were designing the new PSB for, were located in different geographic locations. Due to limited interaction with the user group, we ensured we had a continuous dialogue with the domain experts to familiarize ourselves with the users' behaviour. With this knowledge, we created user persona of the production planner that outlined his roles and responsibilities, needs and goals. The key finding from the customers' requirement was that of 'time as a crucial factor'.

\section{DESIGN}

The next task for us was to come up with a solution which would be based on SAP's frontend library, UI5. We explored various controls UI5 offered and realized the best way to approach the design problem is by incorporating the Gantt Chart control. The Gantt chart enables representation of time dependent data in a graphical fashion. In the initial stage of the project, the Gantt chart control was in its infancy and we had to design workarounds to achieve crucial functionalities. In addition to this, we also had to strictly follow the SAP Fiori guidelines which called in for several constraints.

The old PSB app had too many data elements appearing on one single view. Hence, in the first version of the application, we strictly followed the Fiori guidelines, and tried representing simplicity that Fiori focuses on, by showing minimal and only crucial data upfront to the users [10]. In the cases where the information was not critical for the user, information was shown only on demand, through dialog boxes and popups. The minimalistic data representation, was hence driven heavily through visualizations using colours and shapes.

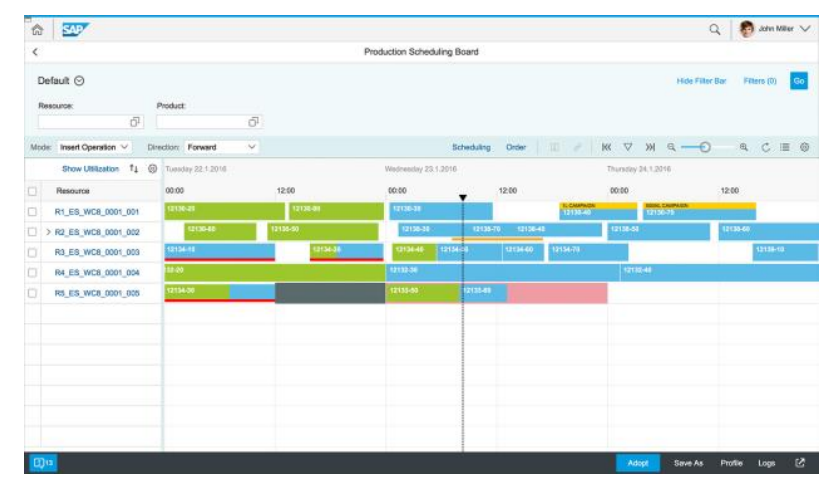

Figure 2: Redesigned 'minimalistic' Production Scheduling Board

\section{TEST}

We conducted usability tests with the customers on this version of PSB application. The tests were conducted in a usability lab with seven users where each user was assigned a moderator. The moderator provided each user with five tasks that had to be completed within a limit of one hour. The users' behaviour while interacting with the application was then observed. The moderator provided clues to the user in case he got stuck while executing any of these tasks.

\section{OBSERVATION}

Initially, most of the users took as much as fifteen minutes to understand the interface. But on reaching the second or third task, they were able to navigate through the application and could find what they were looking for. The time taken to complete each of these tasks was then reduced to less than 5 minutes. At times the users got stuck in performing the tasks and had to be provided with clues. In order to understand the meaning of the colours used in the Gantt Chart, we observed that the users had to continuously refer to the legends which increased the time taken to execute the tasks. While executing the tasks, the users also found dialog boxes as an occlusion to the Gantt view.

More than half the users who had been using the old PSB app, searched for the functionalities similar to the old PSB and tried using the commands that they were comfortable with. We observed that the users had difficulty in interacting with the minimal interface design of the new app due to multiple levels of the information hierarchy that resulted in multiple interactions to find relevant information. This learning curve was steeper for the users who were 
already well-versed with the old PSB application. These users found it challenging to adjust to the new interface that had minimal information upfront as they were conditioned to view a lot of information at a glance which was both crucial and non-crucial.

What we also observed was that the users were not able to quickly distinguish between the graphical elements that were represented with distinct colours. This indicated that they were looking for stronger visual cues.

The finding from these usability tests was that for customers all the data should be visible in one go regardless of how extraneous it is. Additionally, it was also found that the SAP GUI (older) version of PSB, which though seemed complex visually, provided 'good' usability.

This provided us with the insight that simplicity does not necessarily mean minimalism or reducing visual complexity. The perceived visual complexity of displaying maximum data upfront was actually simplicity of usage for the customers [6].

\section{REDESIGN AND IMPACT}

The tests highlighted usability issues that included showing more information upfront, better visualisation of the elements in the Gantt Chart, usage of context menus and tooltips, etc. With these learnings, we redesigned the application by focussing on simplicity in terms of both visual appeal and usability.

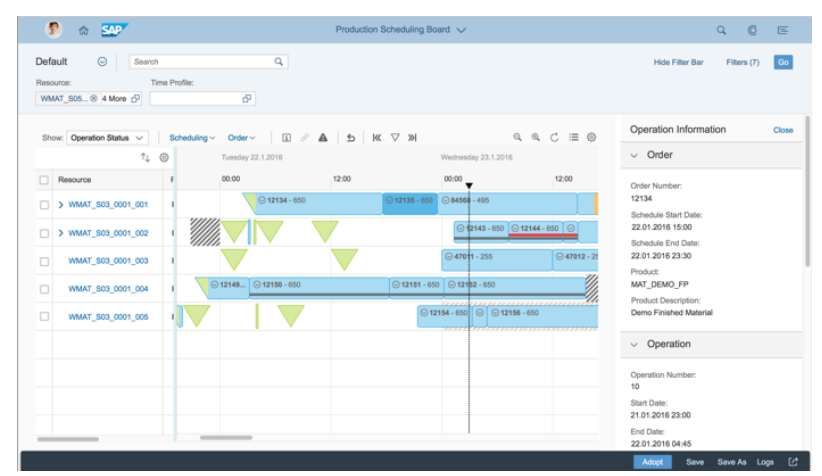

Figure 3: Redesigned Production Scheduling Board after usability test

\subsection{Usage of more colours}

The Fiori UX guidelines had colour palettes with limited colours owing to simplicity. But from the usability tests, we found that introduction of more colours in the application made it more usable for the users to map each of the attributes (such as material, activity, load) to a distinct colour. As a result, we were provided an exception to use more colours in the app.

\subsection{Usage of different shapes}

Initially, the various order types on the Gantt chart of the earlier version of the application, were represented in minimalistic fashion by utilizing only one colour. These order types were now represented not only with distinct colours but also with distinct shapes (such as triangle and rounded rectangle).

We ensured these shapes had a flavour similar to that of the old PSB, that helped the existing users in quick identification of the graphical elements and thereby reducing their learning curve. For example, in order to maintain the consistency between the visual elements, completed operations were represented as green rectangles. This was similar to the blue rectangles that indicated operations that were yet to be completed. But the existing users did not understand the meaning of green rectangles as they were accustomed to see the inverted triangles that represented completed operations.

\subsection{Usage of patterns}

The application required data to be represented in layers (foreground shown with order types, midground and background that was shown with nonworking time and downtime) that was difficult to achieve just with colours and shapes. Hence, we incorporated various patterns that would reduce the cognitive load for the user while interpreting the data elements.

\subsection{Usage of tooltips}

Since the data shown on the application was overwhelming, it would have been difficult for the user to make meaning of information all the time. Hence, we incorporated tooltips that would help the user to quickly comprehend the data set just by hovering the mouse over them.

Additionally, the usage of shapes, patterns and tooltips to represent various data items in a single view, would also be helpful in making the application accessible for colour-blind users.

\subsection{Usage of panels}

Considering the app had plethora of data items would also have more information. We implemented panels that could be shown on hidden from the screen based on user requirement.

\subsection{Addition of Context Menu}

One of the shortcomings of the PSB was that all the actions such as delete, reschedule, etc, vis-àvis the Gantt chart could be triggered only from the toolbar. This was cumbersome for the users as the actions were not in the close proximity to the selected elements [10]. Hence context menus were introduced to the interface. These menus could easily be invoked with a simple right click on the desired graphical element. 
But considering the PSB app was a browser-based application, context menu proved to be a technical limitation as the right click event coincided with that of the browser menu. Later after follow-up discussions with the users we found out that the users rarely used the browser menu and instead, highly preferred the application context menu.

\section{LEARNINGS}

Initially, in order to keep the interface minimalistic, we provided only the crucial data i.e. the Gantt Chart, upfront. The secondary layers of information were made available to the users via dialog boxes and popups. But this minimalistic approach resulted in users finding it cumbersome to execute tasks only from the dialog box, without referring to the complete Gantt Chart. We learnt that instead of showing information as layers, a flat information hierarchy was a better approach. This resulted in removal of dialog boxes and a side panel to show relevant information, was implemented next to the Gantt chart. Though from a designer's perspective, this meant information overload on the screen, but for the users, it ultimately aided them in quick decision making while executing the tasks. The interface was further made richer by using tooltips and context menu that helped the users in informed and quick decision making.

We also gained an understanding of how crucial it is to design for new and existing users both which led to changes in the visual design such as redesign of shapes of the graphical elements that had a flavour similar to that of the old PSB, expansion of colour palette and addition of patterns and icons that facilitated ease of usage for colourblind users.

\section{CONCLUSION AND IMPLICATIONS:}

Through this study, we found that simplicity does not mean minimalism or reducing the information overload. Instead, simplicity is what the user is most comfortable with [6]. Empirical research has the power to disprove preconceived notions of what constitutes the best and most usable design.

The learnings from PSB influenced the design of future Planning and scheduling applications in SAP.

The philosophy of having more information upfront lead to the application, 'Shop Floor Resource Orchestration' having two Gantt Charts in its design. This application was provided with a positive feedback from the users during its usability tests. The feedback and the solutions proposed for PSB were also integrated into the SAP Fiori guidelines later.

\section{REFERENCES}

[1] Uflacker, M. \& Busse D. (2007) Complexity in Enterprise Applications vs. Simplicity in User Experience. In: Jacko J.A. (eds) HumanComputer Interaction. $\mathrm{HCl}$ Applications and Services. $\mathrm{HCl}$ 2007. Lecture Notes in Computer Science, vol 4553. Springer, Berlin, Heidelberg.

[2] Akiki, P.A. \& Bandara, A.K. \& Yu, Y. (2014) Adaptive Model-Driven User Interface Development Systems, ACM Computing Surveys (CSUR).

[3] Singh, A. \& Wesson, J. (2009) Evaluation criteria for assessing the usability of ERP systems. In Proceedings of the 2009 annual research conference of the South African Institute of Computer Scientists and Information Technologists.

[4] Trier, M. and Richter, A. (2013) "I Can Simply..." - Theorizing Simplicity as A Design Principle and Usage Factor". European Conference on Information Systems, Utrecht

[5] Ede, M. \& Dworman, G. (2016, May) Why Designers Might Want to Redesign Company Processes to Get to Better UX Design: A Case Study. In Proceedings of the $2016 \mathrm{CHI}$ Conference Extended Abstracts on Human Factors in Computing Systems (pp. 840-848). ACM.

[6] Joshi, S.G. (2015) Designing for Experienced Simplicity in International Journal on Advances in Intelligent Systems, vol 8 no 3 \& 4, (pp. 324-338)

[7] Schylström, M. (2013). User experience in ERP system development: An action research project to involve user experience in the everyday work.

[8] Denecken, S. (2017), \#S4HANA 1610 use case series: 9a - Production Planning and Detailed Scheduling - PP/DS (biz view).

https://blogs.sap.com/2017/02/02/s4hana1610-use-case-series-9a-productionplanning-and-detailed-scheduling-ppds-bizview/ (2018)

[9] Unknown Author (unknown year) Scheduling with the Detailed Planning Board. https://help.sap.com/saphelp_scm70/helpdata /en/4c/608da3059a06d4e10000000a15822b/f rameset.htm (2018)

[10] Idler S. (2011) Use Gestalt's Laws to improve your UX.

https://designmodo.com/use-gestalt-laws-toimprove-your-ux/ 